\title{
Design and implementation of the data acquisition system of a large scale network based on four-in-one meters
}

For households, water supply, power supply, pipeline gas and centralized heating are necessaries to maintain the normal life of a family. For Chinese urban families, water, electricity, gas, warm services are often provided by different government utility companies. Aimed at the shortage of the manual measurement of the above mentioned services" consumptions, a new mechanism using network metering devices is proposed in this paper. Based on the general network measurement mechanism and through analysis, this paper proposes using meter communication network as the public communication network of various metering devices, forming wireless mesh network by metering devices, constituting a virtual private network by the power company's network, and accessing to metering network based on SCEP protocol and certificates of devices. Under this new network measurement mechanism, a network routing optimization strategy, a data acquisition protocol based on IPv6 and a mesh network management system are designed, and preliminary experiments have been carried on. Experimental results show that the proposed mechanism can realize functions through the network, including the control of multi-measuring devices, topology discovery, data acquisition, fulfilling the multi-meter data acquisition system via network.

Keywords: Energy internet, four-in-one meters, wireless mesh network, virtual private network, SCEP.

\section{Introduction}

$\mathrm{F}$ or residents, water supply, power supply, pipeline gas and centralized heating are essential in normal life of the family. These social public services may be provided by varied entities in different countries and regions. For Chinese urban families, water, electricity, gas, warm service are usually supplied by different government utility companies [1].

Messrs. Wang Peng, Xiao Xiangning, North China Electric Power University, Beijing 102206, China Electric Power Research Institute, Beijing 100192, Xie Bingshan, School of Electronic Information Engineering, Tianjin University, Tianjin International Engineering Institute, Tianjin University, Tianjin 300072 and Su Xueyuan, State Grid Xiamen Electric Power Supply Company, Xiamen 361004, China.
In order to ensure the accurate measurement of the consumption of various public services and to promote energy conservation and emission reduction, Chinese urban residents are promoted to utilize corresponding meters for measurement of household water, electricity, gas, and warm. However, the management of these industries are separated, and the work is carried out independently according to their own industry management model and technical standards, thus resource sharing cannot be formed and workload of meter data acquisition is great, although there are many similarities in their meter data acquisition mode.

As meters for water, electric energy, gas and warm own different needs in measurement and security, household water, warm and gas meters are generally installed interiorly, while electric energy meters installed in the outdoor. Meter data acquisition personnel has to copy dosage by checking every meter inconveniently, entering and exiting each house. The efficiency of such meter data acquisition method is low, and the labour intensity is large, also may mistakes the data. On the other hand, commuting time is not the same due to different household work and rest time. For instance, it is common that the house owner may be away on a business trip. As a consequence, meter data acquisition personnel often go empty road., affecting the overall meter data acquisition and charging schedule. In addition, as a result of distinct living habits, residents' personalized demands are more and more, as well as consciousness of privacy protection and security, increasing difficulties in data acquisition personnel entering the users' home, influencing the efficiency and service quality of meter data acquisition and charging.

The above problems could be solved by connecting corresponding measuring devices through the communication network, thus realizing automatic data transmission. The utilization of transmission network measurement system can gain timely and reliable access to the users' usage data, also, providing a variety of means for users to pay through remote network. Thus reduces a large number of time cost for users who must go to the relevant company for payment in the traditional way, while for companies the services cost in the traditional way can also be saved. However, as automatic 
meter data acquisition systems of different utility companies operate according to their respective industry management and data collection method, there is a fatal flaw: four different sets of automatic meter data acquisition systems run at the same time within a unit area. Such a measurement model is not only a waste of resources, increasing the cost of construction, but also easily leads to inter system interference. Therefore, in order to solve the problem of residential automatic meter data acquisition, we have to break the barriers of these four kinds of industry management modes and transform these four meter data acquisition problems into one meter data acquisition problem, and establish a complete set of automatic meter data acquisition system to unify their automatic meter data acquisition, charging and management. We design

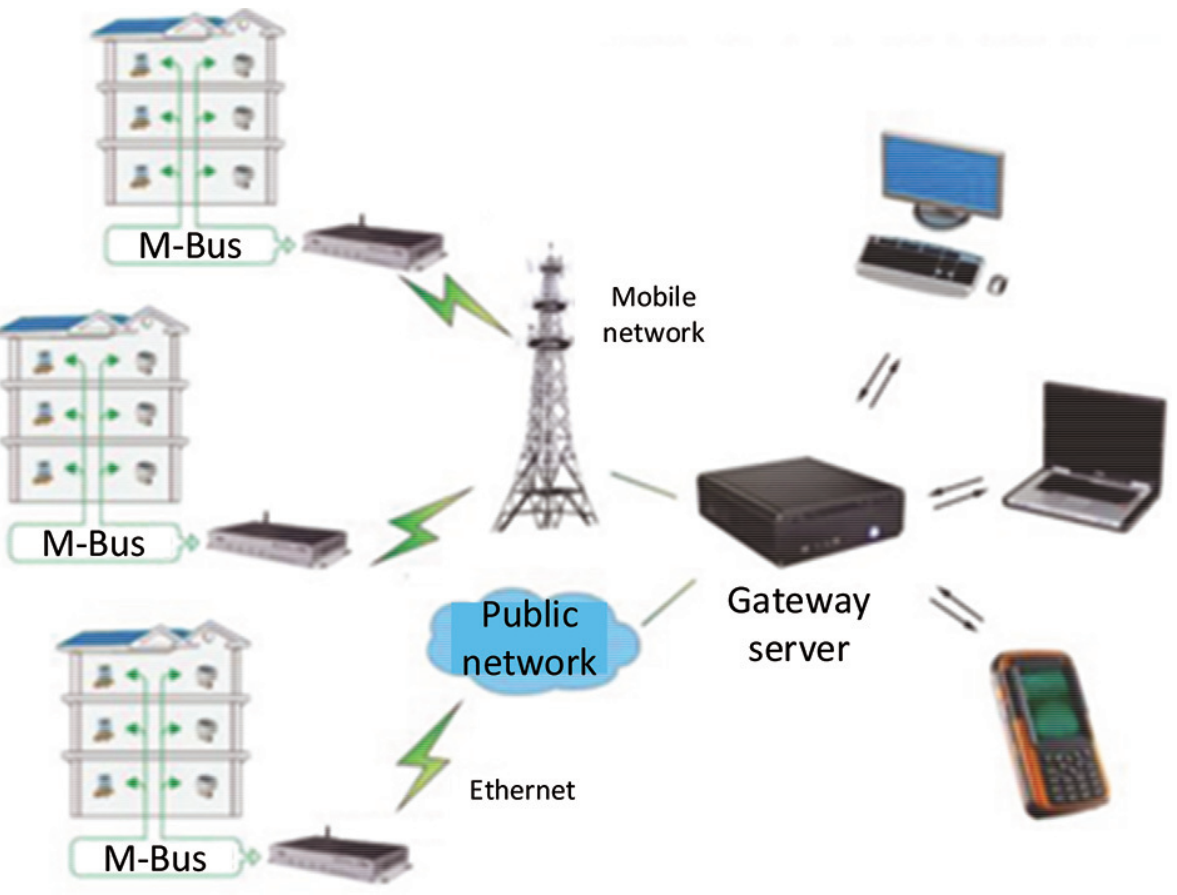

Fig.1 Architecture of the four-in-one measuring network

and implement a network measurement system supporting many utility companies, different metering and remote payments, as well as a high efficiency and reliable network architecture.

\section{System architecture design}

Electricity meters communicate through power line carrier using power lines, thus no need to lay extra lines to connect the entire residents. Therefore, the four-in-one communication channel utilizes the communication channels provided by electricity meter systems. The network meters will upload the collected water, electricity, gas and heating data to their respective administrative departments; these departments will also transport their commands through the networked electricity measurement system to their respective measurement meters. This acquisition and management mechanism break barriers of the separate management among water, electricity, gas and warm.

Since that the majority of the meters support the RS-485 interface or M-bus interface, the M-bus is adopted as the unified measurement procedures interface. 6LoWPAN, a wireless low-power network, is used for networking different meters within a family. Then, wireless meshes are utilized for network construction of multiple families' electrical networks. And next, access to the networked measurement system of the power company is achieved through electric meters with power line carrier or wireless broadband. The architecture is illustrated in Fig.1.

AMI is an integration of many information technologies, including wired technologies, such as fiber optics, power line communication (PLC); as well as wireless communication technologies, such as mobile technologies (3G, 4G, LET), WiMAX, ZigBee, Wi-Fi[2-5]. Due to different communication standards, there are a lot of heterogeneous networks in AMI, which lead to low efficiency, poor stability and low one-time meter data acquisition success rate. To improve these charistics, a new AMI architecture based on IPv6 is proposed. AMI with IPv6 enables the communication network to run on TCP/IP protocol, which greatly improves the compatibility [69]. The AMI structure of smart grid is improved to support a variety of metering devices, as shown in Fig.2.

\section{Security architecture and protocol design}

Due to the demand of establishing a networked measurement system for a number of metering devices of different public service enterprises in a single network, a typical requirement is that different management entities should establish their own virtual network and certification center to achieve safety and independent management.

802.1x IEEE is a port based access control protocol, which is widely used to solve the problem of wireless local area network access authentication. In the process of authentication, all information is encapsulated by the extensible authentication protocol (authentication protocol extensible, EAP) to ensure the security of information transmission on the network. It achieves access control through port switch. Before passing the authentication, the user can only connect with the server through an open and non-controlled port without access restrictions, and cannot access other resources in the network except for 


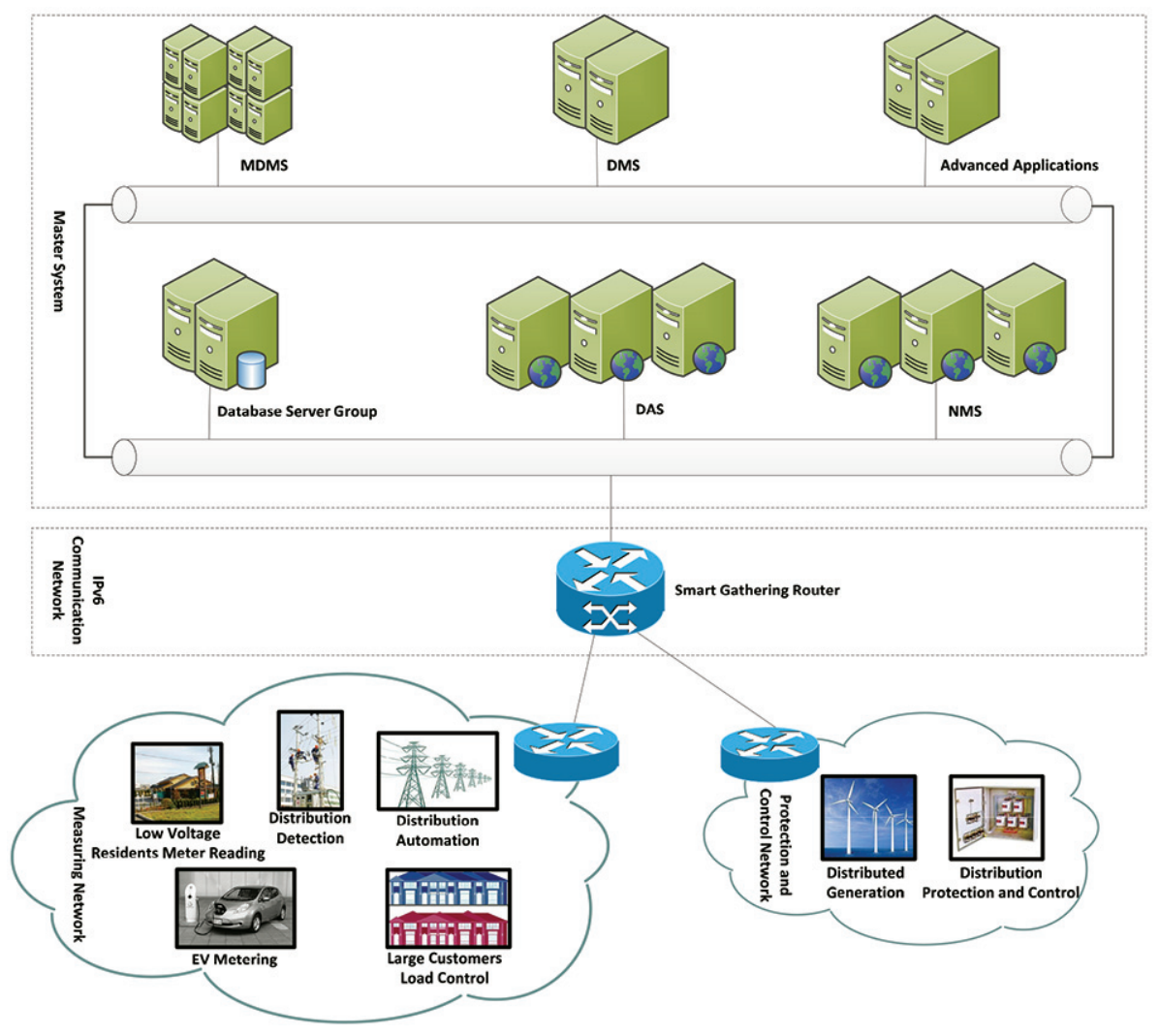

Fig.2 Integrated architecture of smart metering based on AMI key together with the digital certificate generated in an end entity, and then uses the PKCS\#7 protocol to encrypt the communication. In addition, the SCEP certificate registration process is completed online, allowing for automatic registration of certificate, releasing the administrator from such work. SCEP is widely used in network authentication process.

802.1x IEEE can achieve effective isolation of resources in the wireless network through the control of the port, providing a solution for the isolation of AMI network resources. In this paper, the EAP protocol in the 802.1x IEEE protocol is improved, posing the utilization of SCEP to replace the original EAP authentication method, to meet the new certification requirements in the four-in-one networked measurement system. Not only the long-term fixed access smart grid terminals' (such as smart meters, etc.) certification requirements are taken into account, but also the temporary access to mobile smart grid

authentication. After the user successfully pass the authentication, the server sets up a logical channel on the controlled port. The user can access the network resources through the logical channel.

AMI is a wireless sensor network, which can support the 802.1x IEEE protocol to authenticate the access equipment. In the 802.1x IEEE protocol, it is EAP that plays a decisive role in the authentication method. However, existing EAP authentication methods are not suitable for AMI system. EAP-MD5 (Message-Digest5) is a one-way authentication method, and thus cannot provide the client and server mutual authentication. EAP-TLS (Level Security TransPort) is an authentication method based on two-way certificate. However, it requires complex operations such as four handshakes and so on in the process of certification. In addition, EAP-TLS needs to install and maintain certificates at both ends of the client and server, imposing a heavy workload on the administrator. There are a lot of studies on IEEE 802.1x authentication, for example, Park K.Y. et al in [10] analysed the IEEE $802.1 \mathrm{x}$ protocol for mobile routing in wireless local area network security vulnerabilities, and put forward the trusted platform module to solve this problem. K $\mathrm{H}$ Chi et al. in [11] proposed a Fast Handoff security architecture for wireless mesh networks.

SCEP is a certificate-based authentication protocol developed by Cisco, which binds user identity, and the public terminals (such as smart electric vehicles) certification requirements are considered. Furthermore, this article designs a concrete network security architecture which may be used for commerce directly according to the proposed authentication method. In a word, based on the IEEE 802.1x SCEP protocol, this paper replaces the original EAP authentication method with SCEP, and design a detailed network security and authentication architecture, which may be directly used commercially.

The proposed design authenticates users through SCEP, and assigns the metering devices and network devices of different utilities to a group of independent virtual networks which are independent of other enterprises. As shown in Fig.3. The mesh node is consisted of a plurality of electric energy meters, composing the network transmission system of the four-in-one meter data acquisition system. Different measurement meters access the communication network via electricity meters, and then access different virtual networks by their SCEP certificates. These different virtual networks access distinct IPv6 tunnels to access diverse access networks, realizing the isolation and security access of the virtual networks.

The extended 802.1x IEEE protocol authentication method based on SCEP protocol works as the secure authentication method in the security architecture proposed in this paper, in which measurement terminals, including various meters, are 


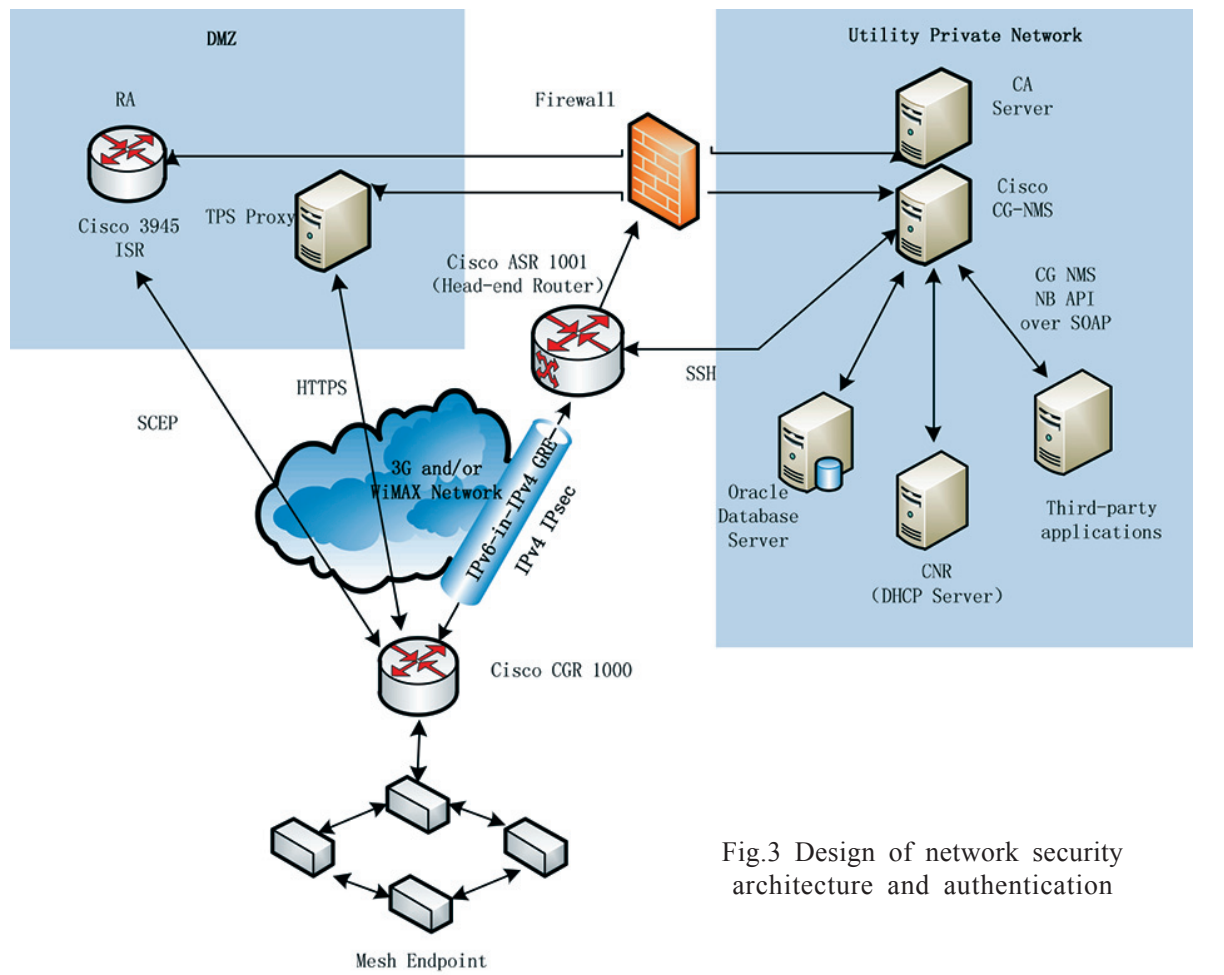

acquisition and certificate registration. Fig.4 illustrates the flow chart of the SCEP-based extended 802.1x IEEE protocol authentication method, taking the smart meter for example:

1. A smart meter sends access request to the convergence router, and then the convergence router returns to the smart meter a request to upload its ID and public key PK.

2. The smart meter sends certification issue request, and attaches its own PK, ID, and a self-signed certificate (Certificate Self-Signed, SSC) generated according to the ID and the PK.

3. CA/RA server receives the certificate issue request forwarded by the convergence router, and verifies the meter's SSC using PK, and then issues a certificate if successfully verified. At the same

the end entities of the SCEP protocol. The RA is router with high processing capacity, to deal with applications for SCEP certifications submitted by the network measurement terminals. The CA authentication server is placed behind a firewall. And thus the firewall filters source IP address, destination IP address and the data protocol to ensure that CA server only receives packets with allowed IP addresses from network terminals and SCEP protocols, reducing the risk of CA server being intruded.

Extended 802.1x IEEE protocol authentication method is expanded mainly using processes of certificate authority and certificate registration occurring in SCEP protocol. This authentication method is more applicable to the certification system with a variety of measurements of different management institutions. According to the security authentication method designed in this paper, the aggregation router provides the function of AP in the $802.1 \mathrm{x}$ IEEE protocol, instead of measurement terminals sending the access network request, the SCEP certificate issue request and the certificate registration request. On the other hand, certificate query and certificate revocation of measuring terminals are not extended to the IEEE 802.1x protocol, keeping in consistent with original SCEP protocol. In this scheme the implementation is described as follow: convergence router will send SCEP certificate query request and revocation request from intelligent terminal to the frontend router, and then the front-end router forwards them to CA and LDAP server, so as to realize the two functions.

How the proposed design differs from the original SCEP protocol will be discussed below, that are certificate time, CA encrypts the ID and the certificate requests operation number in meter's SSC by PK, and then sends the cipher text back to the smart electricity meter.

4. After receiving the $\mathrm{CA} / \mathrm{RA}$ cipher text forwarded by convergence router, the smart meter decrypts it by the local private key and sends a MD5 fingerprint to CA/RA.

5. CA/RA receives the MD5 fingerprint and performs verification. If the verification is successful, then the certificate will be issued, while the issue will be failed if the certification is not successful.

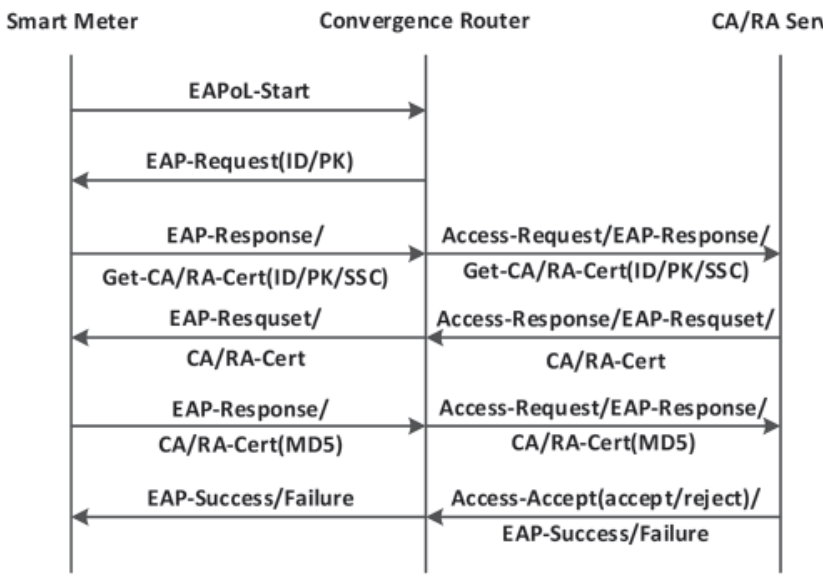

Fig.4 Certificate acquisition flow chart

Certificate registration request is relatively simple. A certificate registration request sent from a smart meter, forwarded by the convergence router to CA/RA, after the consent of the CA/RA, certificate registration is successful. Fig. 5 is the process of the certificate registration. 


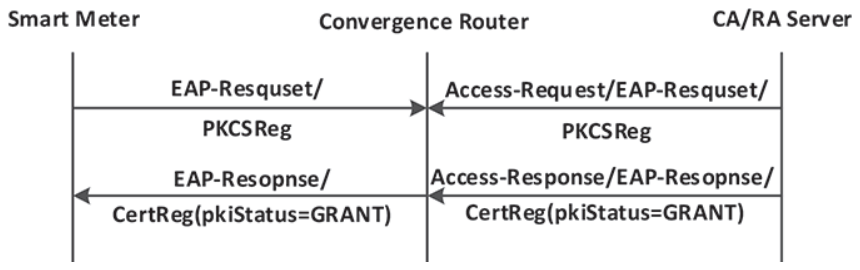

Fig.5 Certificate registration flow chart

\section{Network measurement system design and preliminary experiment}

In the support of the above mentioned virtual private network and large scale IPv6 measuring network, a network measurement management system is designed in order to achieve data acquisition from a variety of meters, identity authentication and remote service purchase and management.

Firstly, optimize energy network routing for large-scale measuring devices and network business; secondly, based on 6LoWPAN, manage mesh network, and manage IPv6 tunnel and virtual network; and finally realize the preliminary prototype management system, performing remote management of metering devices.

\subsection{RouTING ALGORITHM DESIGN BASED ON DEGREE AND WAITING TIME}

This strategy is a local routing strategy, suitable for large scale, highly flexible measuring network [12-14]. It takes into account the effects of both dynamic factors and static factors on routing efficiency, ensuring the maximum efficiency of multi information flow transmission. This strategy considers the characteristics that daily consumption of electricity of various types of users will change with the time, so that it adaptively adjusts parameters, fitting with the actual situation better.

\subsubsection{Strategy overview}

The strategy process includes building a network model, adding new energy information flow, searching the destination node, passing the energy information flow, etc. The flow chart is described as illustrated in Fig.6:

1. Establish network model. As energy internet is the combination of the Internet and renewable energy [15-17], energy Internet can be considered to have the same network structure with the Internet. And the Internet is a typical scale-free network, in order to ensure the general case, this paper adopts the very classic BA network model proposed by Barabási and Albert for simulation [18].

2. Add new energy information flow. Starting from no load, add R (T) energy information flow to the network each time step, and each energy information flow randomly selected source and destination nodes. Take it into consideration that the actual electricity consumption will appear peak and valley alternately, so the $\mathrm{R}(\mathrm{T})$ values change over time. The period and the value refer to the

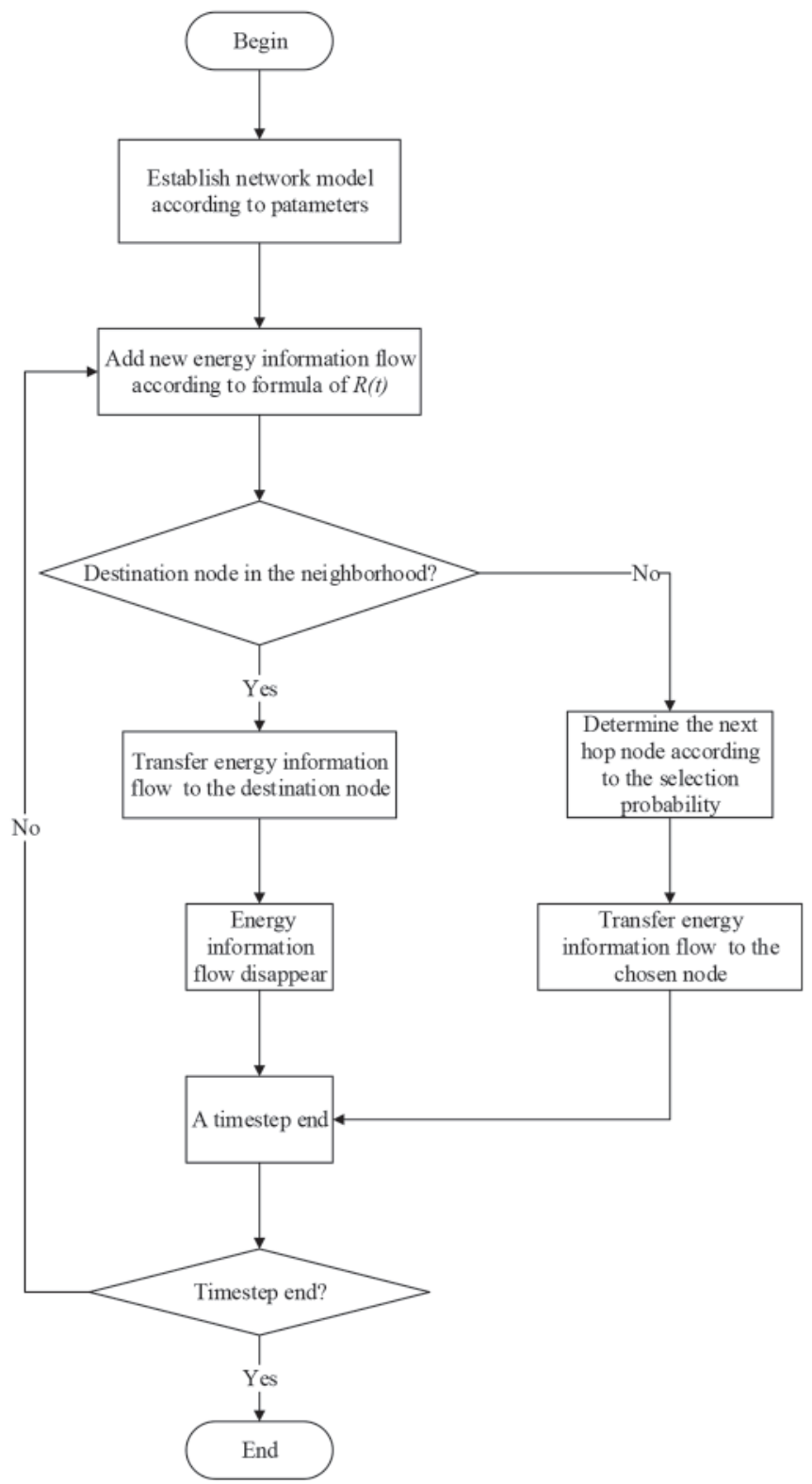

Fig.6 Routing strategy schematic

electricity peak valley division and charges method of time price in China, specifically: peak period 8 hours (8:00 to $11: 00,18: 00$ to $23: 00$ ), flat section 8 hours (7:00 to 8:00, 11:00 to $18: 00)$, valley section 8 hours (23:00 to 7:00). Peak tariff is $150 \%$ of flat price, while valley price is $50 \%$ of flat price. $\mathrm{R}(\mathrm{T})$ changes with time as expressed in Equation (1):

$R(t)= \begin{cases}30 & 8<t \% T<11,18<t \% T<23 \\ 20 & 7<t \% T<8,11<t \% T<18 \\ 10 & t \% T=23,0<t \% T<7\end{cases}$

where $\%$ denotes residual operation, $T=24$. Newly generated energy information flow will be added to the tail of the source node queue. Each node's queue contains energy information flow both generated by itself and 
transported from other nodes.

3. Search for the destination node. Each node processes according to the first in first out (FIFO) and the path interation avoidance (PIA) rules in the transmission of energy information flow [14]. First, search its neighbors. If there is a destination node in the neighborhood, the energy information flow will be transported directly to the destination node; if any of the neighbors is not the destination node, next node is selected in accordance with the selection probability of each node. The probability calculation method will be analyzed in the next section.

4. Transfer energy information flow. The energy information flow will be transferred to the selected node, and then added to the tail of the node queue. The energy information flow will disappear in the network after it arrives at the destination node.

The following part details the core of this paper: the determination of transmission probability between neighbor nodes in information flow transmission.

\subsubsection{Transmission probability calculation based on degree and waiting time}

As mentioned above, a global routing strategy has good performance, but requires that each energy flow knows the topology of the entire network, which is obviously not suitable for large-scale networks. While although local routing strategy performances slightly inferior, transfer occur just needs to know the topology information of neighbors. The calculation of the mentioned transmission probability in the third step can generally be divided into two sorts: staticinformation-based algorithm and dynamic-information-based algorithm.

\section{A. Static-information-based algorithm}

In the local routing strategy, the static information refers to the distribution of the degree of each node. In general, a router with higher degree is a router with greater transmission capacity, and is more important. Therefore, the node transmission probability depends on the degree of this node. The basic calculation of transmission probability based on degree is expressed as follows:

$$
P_{i \rightarrow j}=\frac{k_{j}}{\sum_{\log (i)} k_{l}}
$$

where $P_{i \rightarrow j} i$ is the probability of energy information flow transmitted from node $i$ to node $j, k_{j}$ represents the degree of node $j, g(i)$ represents the set of neighbors of node $i$. It can be seen from equation (2) that the greater degree of a node is, the bigger will be the probability of transmission occurrence.

\section{B. Dynamic-information-based algorithm}

In local routing strategy, dynamic information may refer to the length of the queue in the energy information flow of each node at a time $[19,20]$. However, considering the different transmission capacity of nodes, nodes with bigger transmission capability tend not to congest even if the queue is longer. Therefore, in this article, dynamic information is considered as the waiting time between an energy information flow arriving at a node and being transferred again. It is calculated by:

$$
t_{j}=\frac{q_{j}}{c_{j}}
$$

where, $t_{j}$ is the time an energy information flow waiting at node $j . q_{j}$ is the queue size of node when energy information flow is being transmitted, changing with time. $C_{j}$ is the transmission capability of node $j$.

The basic calculation of transmission probability based on waiting time can be expressed as:

$$
P_{i \rightarrow j}=\frac{e^{-t_{j}}}{\sum_{l \in g(i)} e^{-t_{l}}}
$$

where $e^{-t_{j}}$ is attenuation term. It is obvious that if a node owns smaller $t_{j}$, then it has lagger $e^{-t_{j}}$, and thus lager transmission probability.

\section{Degree-and-waiting-time-based algorithm}

From the discussion of the above two sections, it can be known that the degree and energy information waiting time of a node both have effects on routing efficiency. Therefore, we propose a transmission probability calculation method based on the combination of degree and waiting time, tuning the weight of these two through the adjustment of a parameter $\alpha$. The transmission probability is calculated by:

$$
P_{i \rightarrow j}=\frac{k_{j}^{\alpha_{e}-(1-\alpha) t_{j}}}{\sum_{l \in g(i)} k_{l}^{\alpha_{e}-(1-\alpha) t_{l}}}
$$

where $\alpha$ is a tuning variable with value range $[0,1]$. It determines the importance of degree and waiting time in the chosen of energy information flow routing process. When $\alpha$ is lager, energy information flow has a higher probability to choose neighbour nodes with higher degree. While when $\alpha$ is smaller, energy information flow tends to choose neighbor nodes with lower degree. When $\alpha=0$, equation (5) becomes static-information-based algorithm.

Considering the time energy information flow stays in the net reflects scheduling efficiency of energy, more important, the quality of the routing strategy. Therefore, takes the average time $\langle T\rangle$ that energy information flow exists in the net as an evaluation index. The smaller the $\langle\mathrm{T}\rangle$ is, better will be the routing strategy, $\langle\mathrm{T}\rangle$ is calculated according to:

$$
\langle T\rangle=\frac{1}{A} \sum_{i=1}^{A} T_{i}
$$

where $A$ is the total number of energy information flow 


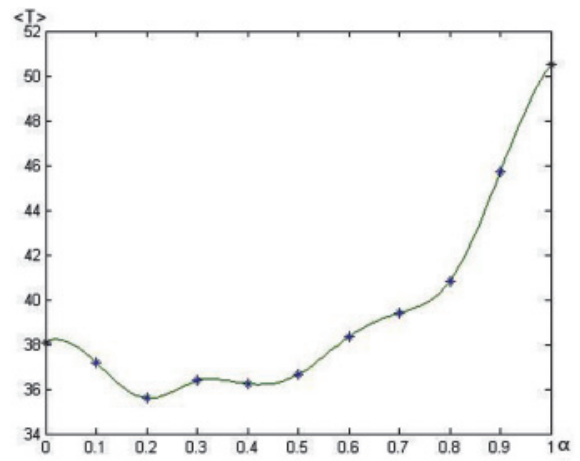

(a)

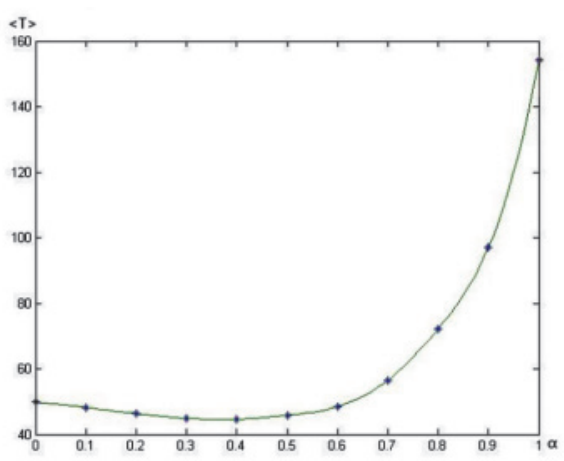

(b)

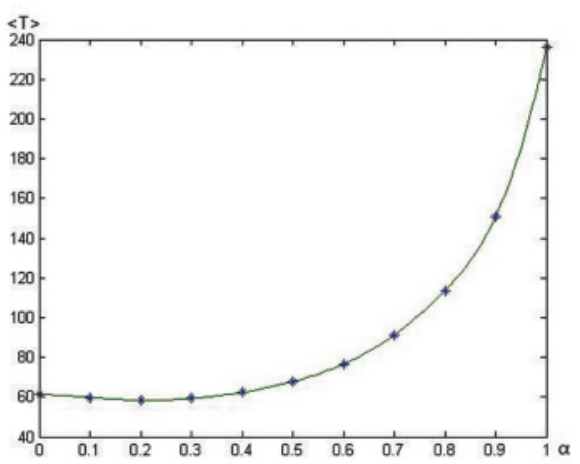

(c)

Fig.7 Relationship between and $\alpha$ with different $R$

generated within a given period of time. $T_{i}$ denotes the time that a single piece of information flow transfers from source node to destination node spends, including the transmission time and the waiting time on the congested node. Obviously if the network model and the routing strategy are determined, the size of $\langle\mathrm{T}\rangle$ only depends on $\alpha$ and $R(t)$, namely .

For a routing strategy of this kind, usually an optimal $\alpha$ can be found, such that the transmission efficiency is the highest. However, due to that the actual value of $R(T)$ will change over time, each value of $R(T)$ corresponds to a $\alpha$ which gets the optimal transmission efficiency. So before the execution of the strategy, firstly, we traverse $\alpha$ to calculate the optimal $\alpha$ under a fixed $R$ value to gain the highest transmission efficiencies, denoted as $\alpha_{\min }$. Then during the implementation of the strategy, parameter $\alpha$ is adaptive to $R(t)$, achieving the optimal transmission efficiency.

\subsubsection{Routing algorithm performance simulation}

\section{A. Network model and simulation overview}

The BA network model is adopted for the simulation. In the model, node degree distribution $p(k)$ has a power-law characteristic, i.e. $p(k) \sim k^{-v}$, with $\gamma=3$. Make model initial nodes number $\mathrm{m} 0=3$, and increase node number by $\mathrm{m}=3$ for each loop. The final nodes number is $\mathrm{N}=1000$. The transmission capacity of each node equals to the degree of the node, namely $C_{i}=k_{i}$. Simulation is based on win7 system, R2014a MATLAB version.

Simulation is divided into the following steps:

1. Take different values of $R$, and calculate corresponding $\alpha_{\min }$. Set R to 10, 20, 30 separately, and then transverse $\alpha$ within the range of $[0,1]$ with step 0.1 to find the corresponding $\alpha_{\min }$, and compare whether they are same and change as $\mathrm{R}$ varies.

2. Take different values of $\alpha$, and make comparison of $\langle\mathrm{T}\rangle$ with same time step and different conditions. When considering influence of degree only, it can be conducted that $\alpha=1$, while when considering waiting time only, the result is $\alpha=0$; when both factors are considered, form equation (1), it can be seen that $\overline{R(t)}=20$. Therefore, make $\alpha$ the value of corresponding $\alpha_{\min }$ when $R=20$, representing the circumstance that $\alpha$ does not adapt to $R(t)$; when $R(t)$ varies, make $\alpha$ take the value of corresponding $\alpha_{\text {min }}$, denoted as $\alpha$ mix, representing the transmission probability proposed in this paper.

B. Simulation results and analysis

Fig. 7 shows that the traversal $\alpha$ of different values of $R$, in which (a) (b) (c) corresponds to $R=10, R=20, R=30$, respectively. Each subfigures in Fig. 7 plots the relationship between $\langle\mathrm{T}\rangle$ and $\alpha$. Fig.7(a) shows all the simulation results, while Fig.7(b) shows part of the results after amplification, taking values of integer multiples of $t=480$. The curve in the graph is obtained by spline interpolation function.

From Fig.7, when $R=10$, it can be seen that $\alpha_{\min }=0.2$ while $\alpha_{\text {min }}=0.4$ when $R=20$ and $\alpha_{\text {min }}=0.2$ when $R=30$. It can also get three conclusions:

1. $\langle\mathrm{T}\rangle$ gets minimum value at neither $\alpha=0$ nor $\alpha=1$, denoting that considering only dynamic or static information will not be the optimal routing strategy, both leading to prolonged transmission of energy information flow. Only taking into account two factors and select the appropriate parameters can result in maximum transmission efficiency.

2. $\alpha_{\text {min }}$ varies as $R$ varies, denoting that different $R$ corresponds to different $\alpha_{\text {min }}$, thus the routing strategy proposed in this paper is reasonable and feasible.

3. Compare the curves of the two sides of $\alpha_{\min }$, it can be observed that the value of $\langle\mathrm{T}\rangle$ with $\alpha$ close to 1 is significantly greater than that with $\alpha$ close to 0 , denoting that the dynamic information in the routing strategy occupies a relatively important position.

Fig.8 illustrates the relationship between $\langle\mathrm{T}\rangle$ and $\mathrm{t}$ under different $\alpha$ values. Fig. 8 (a) shows that, with the extension of time, when $\alpha=1,\langle\mathrm{~T}\rangle$ value increased significantly, while in other cases, the increase is obviously slowed down after $\langle\mathrm{T}\rangle$ reaches around 50, and has little difference from others. In order to observe the $\langle\mathrm{T}\rangle$ curves of these three cases more clearly, this part of the curves is zoomed in, and the result is shown in Fig.8(b). From Fig.8(b), we can see that 
$\langle\mathrm{T}\rangle_{\alpha=0}>\langle\mathrm{T}\rangle_{\alpha=0.4}>\langle\mathrm{T}\rangle_{\alpha m i x}$. When $\alpha=0$ and $\alpha=0.4,\langle\mathrm{~T}\rangle$ increases gradually, and the curve slope of the former is greater than that of the latter, and when $\alpha=$ mix, the curve is basically stable, and the $\langle\mathrm{T}\rangle$ variance is small. The gap between the three curves increases gradually with time extending. Therefore, it can be concluded that the routing strategy proposed in this paper has a great improvement compared to the pure static routing strategy, as well as advantages over the pure dynamic routing strategy, which will gradually increase over time. And for the routing strategy itself, a changing $\alpha$ which is adapt to $R$ performs better than a single static $\alpha$.
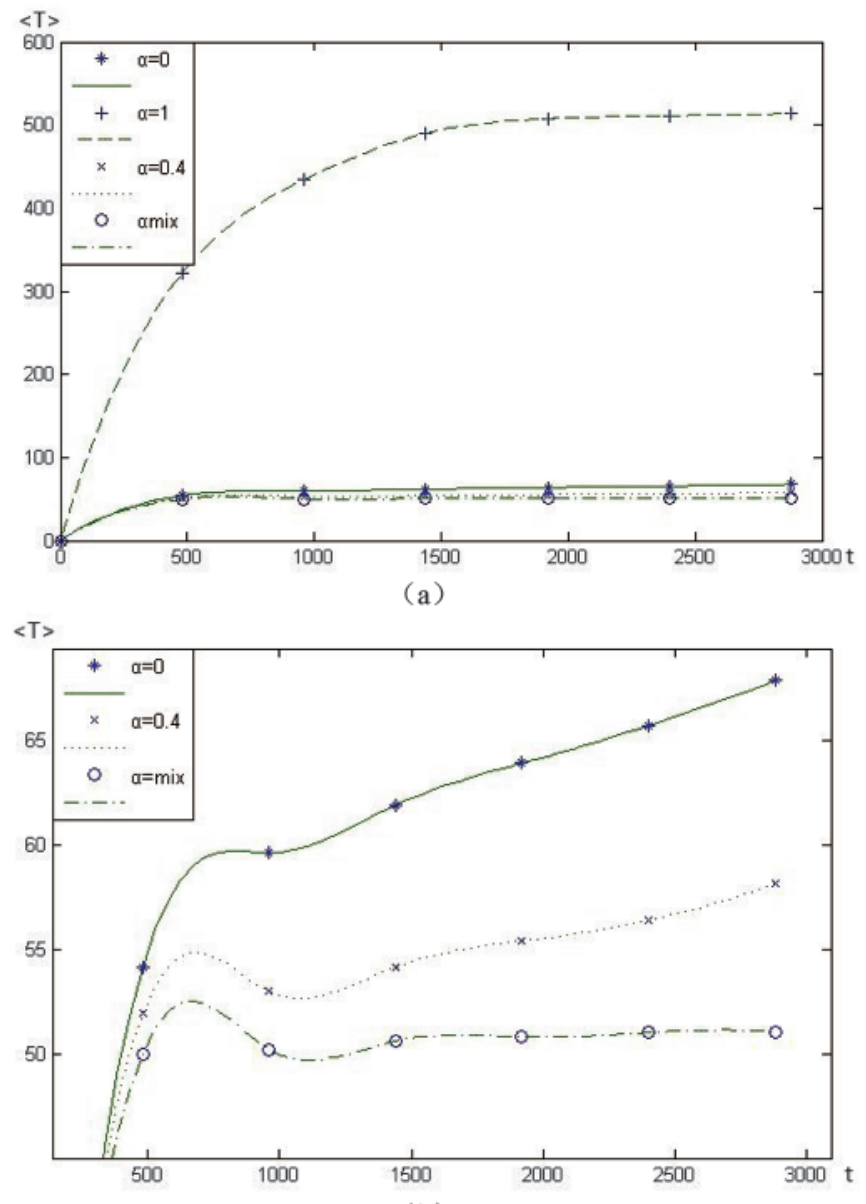

(b)

Fig.8 Relationship between $\langle\mathrm{T}\rangle$ and $t$ under different $\alpha$ values

4.2 FOUR-IN-ONE-NETWORKED-METER MEASUREMENT SYSTEM DESIGN

\subsubsection{Establishment of a new network model}

In the process of implementation, to ensure communication ability and reduce cost, different wireless and wired network technologies are analyzed, and the technology which combines Zigbee and Wifi, and IPv4 and IPV6 is proposed. ZigBee technology has a successful application in electricity energy information collection and management system. ZigBee alliance provides the ZigBee Smart Energy
Profile protocol set and interoperability standards. 802.11 technology is the mainstream technology of WLAN, featuring in long communication distance. It can constitute the mesh network, and expand the network coverage.

Through the analysis and comparison of the transmission distance, application costs, and network's physical transmission capacity which is needed in meter information collection and status estimation, of the above wireless network technologies, the comprehensive utilization of ZigBee and IEEE 802.11 technology is proposed, and establishes a new network model in which three levels access topology is formed with the backbone network.

TAble 1: Three-TIER ACCESS Network

\begin{tabular}{lccc}
\hline Parameters & Meter & $\begin{array}{c}\text { Primary } \\
\text { concentrator }\end{array}$ & $\begin{array}{c}\text { Backbone } \\
\text { concentrator }\end{array}$ \\
\hline Wireless hardware & ZigBee & ZigBee+802 & $\begin{array}{c}802+\text { fixed } \\
\text { network }\end{array}$ \\
Network protocol & IPv4 & IPv4 & IPv4+v6 \\
Position/placement & $\begin{array}{c}\text { Every } \\
\text { househood }\end{array}$ & $\begin{array}{c}\text { Each 4/5 } \\
\text { layer }\end{array}$ & $\begin{array}{c}\text { Each } \\
\text { substation/cell }\end{array}$ \\
\hline
\end{tabular}

The new network access structure will widely install low cost ZigBee communication module in residential electricity meters, for short distance data collection, remote control and management. As the ZigBee signal transmission occlusion within buildings, network coordinators (work as the primary data concentrator) supporting ZigBee and 802.11 protocols need to be placed in some residents' smart meters or intelligent ammeter boxes outdoor. As the shielding effect of floors in the building for wireless signal is more serious, it is difficult for ZigBee communication module to transmit directly to data concentrator which is away with an interval of five floors. Therefore, an 802.11 primary concentrator is deployed every five layers. Taking into account that there are two ways for a concentrator to connect to the backbone network, so their location need to be carefully considered. For 802.11 concentrators accessing directly to the wired backbone, the location should be convenient for network construction. For those need other 802.11 access concentrators or relays to access the backbone, the location should has less wireless signal interference and different non-overlapping channels (for example $802.11 \mathrm{~b}$ in 1, 6, 11 is non-overlapping channels) with WLAN device channels used by nearby residents, as far as possible. Thus ensure the interconnection of multiple primary 802.11 data concentrators, also avoid the interference of the wireless network for home users.

Such a multi access mode has the following advantages: (1) Primary concentrators become the gateway of metering device communication net and backbone concentrator communication net. It enables the isolation of communication module or interface of the metering device, and backbone. Thus the changes will not influence each other. (2) Different devices use different network technologies, thus adjust measures to local conditions, reducing the overall cost. 
Still take the example of a medium-scale residential cell with 5000 households. It has $1010 \mathrm{kV}$ substations and each substation access to 500 users. The cell has 50 business users. Suppose that the 50 business users are in a separate two-story building, and the 500 households are distributed in five 25-story high-rise buildings with 100 households in each one. In accordance with the above mentioned network architecture and implementation recommendations, the required network equipment includes:

50 Business uses: one ZigBee module for every household, and a primary concentrator for all.

Each high-rise building (100 households): a ZigBee module for every household, 5 primary concentrators $(25$ layer $/ 5=5$ ).

The whole cell accesses a backbone concentrator.

Totally, 5050 ZigBee communication modules, six primary concentrators, one backbone concentrator are needed. The price of Zigbee modules is about tens of yuan, while that of primary concentrator is thousands of yuan, backbone concentrator ten thousands of yuan.

Therefore, the communication equipment cost of the above network is about 20 30 million. The designed service life of the equipment is eight to ten years and equipment cost is only two to three million yuan each year, household equipment cost is as low as tens of yuan, far less than the cost of manual meter data acquisition. And the real-time load data and reliability data of grid can be obtained, and realized remote load management and control.

\subsubsection{Design and implement a multi-meter-network acquisition protocol based on SNMPv6}

After the above access network is constructed, an efficient and reliable intelligent meter data transmission protocol is necessary for the real-time transmission of meter state, electricity information, etc. Currently there is no unified electricity energy meter communication protocol specifications, mainstream meter specification is the DL/T 645 and its various extended version. The system specifications includes Zhejiang (Guangdong) system specifications, the national electric system specifications, Shanghai system specification, DL/T 719-2000 (IEC 60870-5-102) and the derived versions of 102 statute, etc. The emergence of international standard IEC 62056 "the electric energy metering - data exchange of meter data acquisition, tariff and load control "series solved the above problems. It defines object identifier (OBSI) from the perspective of communication, and establishes interface model of instruments, unifying communication protocol based on open system interconnection model - device language message specification (DLMS). Therefore, collect information using
IEC62056 as the basis and standard is reasonable and feasible. Considering that there will be a larger number of meters, IPv6 protocol will be utilized in the future. Therefore, the design of the protocol of data acquisition and management will use IPv6 protocol and develope on the basis of SNMPv6 protocol.

The basic idea is to consider all meters and concentrators as network equipment and utilize management mechanism to collect information, and the meters can initiatively inform the concentrator when various events occur. The specific method is: send polling measurement information at regular intervals; send the state changes, the possible fault information and other abnormal information as trap.

Through the analysis of the nature and function of meter data, it can be known that the reliabilities and priorities of different kinds of data are different. This makes it possible to use the network transmission capacity reasonably, and reduce the number of trap, and compress the number of packets to send. The key method is trap merging mechanism. The unimportant traps are not sent and processed immediately after generation, but after a predetermined delay. Each PDU includes only one trap message. The format is: time, traps generated within this period of time merging together to send. Thus reduces the number of packets to send, and reduces the occupation of the channel, greatly reducing the impact of data transmission on network resources. General trap message format is shown as in Fig.9.

Where the fields of PDU type and Agent Addr are the same for the same sending device. Thus if transmitted using combined method, only needs to send it once, reducing redundant data occupying the network bandwidth resources. The combined trap packages require a small number of changes, which allows managers to split the original trap message for processing and storage. The method taken is that using original unused field to store information, and modifying the variable bindings to indicate the location of different traps. The main processing procedures are:

1. According to the measurement requirements, dynamically determine data priority, and classify the data in accordance with the priority. Based on the remote control information and rules of the electric energy metering center, the data of smart electric meter collected by the network should be assigned on different priorities. The electricity metering data of fixed intervals is classified as a general priority; the terminal alarm information is classified as important information; the terminal fault and power down state information are classified as emergency information.

2. Transfer the classified measuring device data into SNMP report packet format. First, the packet is compressed by

\begin{tabular}{|l|l|l|l|l|l|l|l|l|l|}
\hline Version & Community & $\begin{array}{c}\text { PDU } \\
\text { Type }\end{array}$ & Enterprise & $\begin{array}{c}\text { Agent } \\
\text { Addr }\end{array}$ & $\begin{array}{c}\text { GEN } \\
\text { Trap }\end{array}$ & $\begin{array}{c}\text { Spec } \\
\text { Trap }\end{array}$ & $\begin{array}{c}\text { Time } \\
\text { Stamp }\end{array}$ & VBS & $\cdots \cdots$ \\
\hline
\end{tabular}

Fig.9 General trap message format 
LZW algorithm, and then the compressed data and priority information are encapsulated together as SNMP reporting message.

3. Adaptive packet merging and transmission, which is further divided into the following steps:

(a) When need to collect measuring devices information through the network, check its priority, if it is a general priority message, start the timer and go to step (3D); otherwise go to step (3B).

(b) Determine whether it is important information. If so, send the message and require the receiver to send receipt, and then return to step (3A). Otherwise go to step (3C).

(c) Send emergency message.

(d) Determine whether the timer times out. If not, turn back to step (3A). If so, merge all the general information into a whole SNMP report message before the timeout is sent. In the traditional trap, each message needs to be sent to the manager. The data part is 86 bytes, and message header information needs extra 50 bytes.

For the cell discussed above, if $5 \%$ of the information needs to send trap, then about 500 thousand trap messages are produced per day. when transmitted directly, the amount of data is: $500000 * 136$ bytes $=68000000$ bytes, about $68 \mathrm{MB}$. Assume that the messages are divided into 3 levels in terms of importance and timeliness requirements, and the proportion is emergency: general: no emergency $=1: 2: 7$. Then the no emergency information merges every five pieces. Thus the no emergency information forms a 960 bytes message, and through the compression of LZW algorithm, the message sent is 320 bytes, actually.

Calculate the amount of data sent by the new data acquisition protocol:

500 thousand packets fall into 50 thousand packets of emergency, 100 thousand packets of general, 350 thousand packets of no emergency which are merged into 70 thousand packets. The total number of packets is reduced from 500 thousand to 220 thousand, and the amount of data transmitted is reduced to about $40 \%$.

\subsection{Preliminary experiment}

This experiment aims at the function expansion and testing of the display of mesh structure network topology. The network topology update and the meter data acquisition are automatically carried out in the background. When the network topology is updated, the automatic acquisition of the meter data is stopped, and is not started again until the network topology update is completed.

The experiment is conducted with ASUS K751L, Windows 8.1. Use Java servlet for development, Tomcat 7.0 server and MySQL database. The initial page of the system is shown in Fig.10.

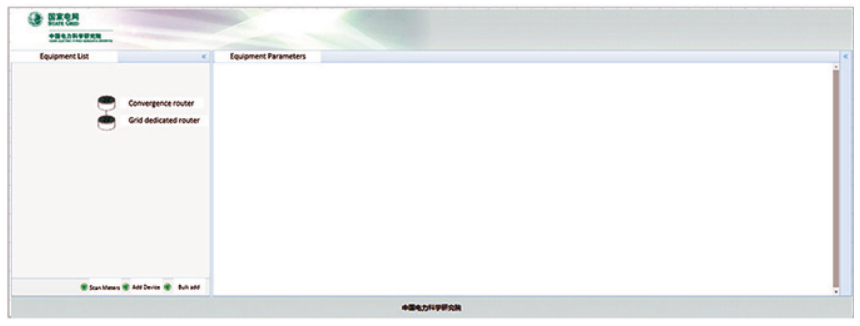

Fig.10 Initial page

Equipment List

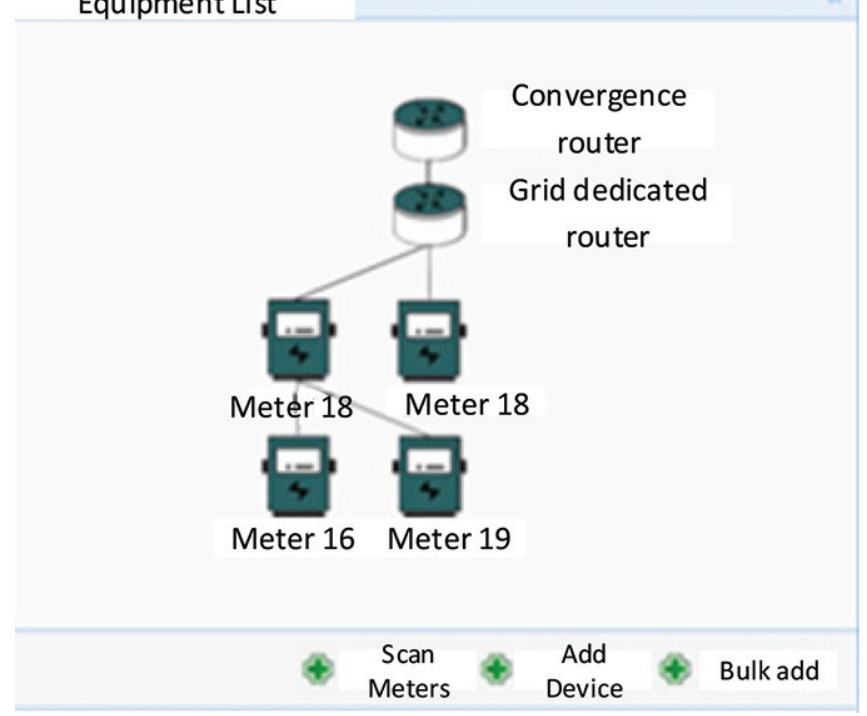

Fig.11 Close-up of the topology

The hierarchical topology of the mesh network composed of several meters is studied. A close-up is shown in Fig.11. Each meter is clickable, and the latest data will be displayed when click on a meter.

When click on any electricity meters in topology, device parameter area will display the latest data, as shown in Fig.12.

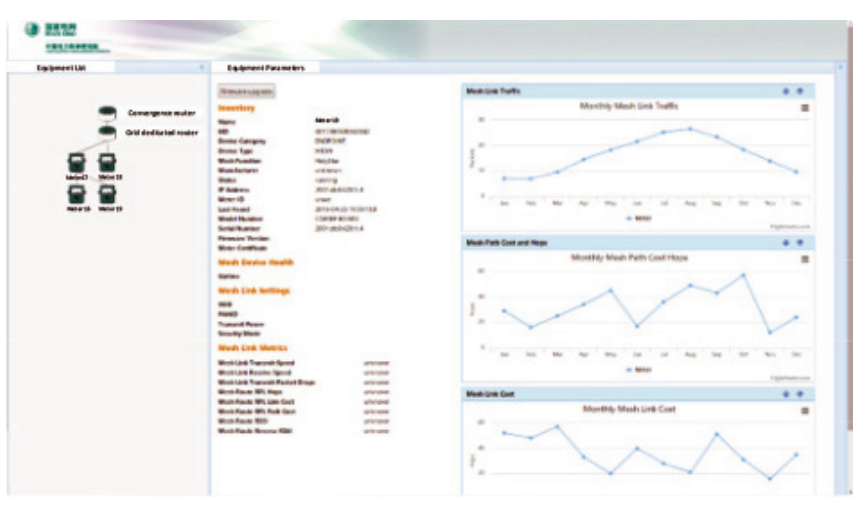

Fig.12 Meter data acquisition

\section{Conclusions}

In this paper, metering needs of a variety of public services such as electricity, water, piped natural gas, central heating are analyzed, and then find the problems in manual measurement. Aiming at the deficiency of the artificial way, a 
new mechanism of using the networked metering devices is put forward. Furthermore, based on the general network measurement mechanism, a detailed method is proposed through analysis, which is utilizing communication network of electricity as the public communication network for various measuring devices, wireless mesh network formed by measuring devices, virtual private networks constituted by the power company's network, and access mode to metering network based on certificate of SCEP protocols and devices.

Under the new networked measuring mechanism, a routing optimization strategy is designed, as well as a data acquisition protocol based on IPv6 and a mesh network management system. At last, a preliminary experiment has been carried out. Experimental results show that the control of multi measurement devices, topology discovery and data acquisition can be realized through the network, realizing the multi-meter networked data acquisition system.

\section{Acknowledgements}

The authors would like to thank the reviewers for their valuable comments and suggestions which have improved the presentation of the paper.

\section{References}

[1] Ministry of Housing and Urban-Rural Development of P. R. China, www.moherd.gov.cn

[2] Yan Y, Qian Y, Sharif H, et al. (2013) : A survey on smart grid communication infrastructures: Motivations, requirements and challenges[J]. Communications Surveys \& Tutorials, IEEE, 15(1): 5-20.

[3] Bian D, Kuzlu M, Pipattanasomporn M, et al. (2014) : Analysis of communication schemes for Advanced Metering Infrastructure (AMI)[C]//PES General Meeting| Conference \& Exposition, 2014 IEEE. IEEE, 1-5.

[4] Erol-Kantarci M, Mouftah H T. (2015): Energy-efficient information and communication infrastructures in the smart grid: A survey on interactions and open issues [J]. Communications Surveys \& Tutorials, IEEE, 17(1): 179-197.

[5] Charni R, Maier M. (2014): Total cost of ownership and risk analysis of collaborative implementation models for integrated fiber-wireless smart grid communications infrastructures[J]. Smart Grid, IEEE Transactions on, 5(5): 2264-2272.

[6] Balakrishna P, Rajagopal K, Swarup K S. (2014): Analysis on AMI system requirements for effective convergence of distribution automation and AMIs[C]/ /Power India International Conference (PIICON), 6th IEEE. IEEE, 2014: 1-7.

[7] Akkaya K, Rabieh K, Mahmoud M, et al. (2015): Customized certificate revocation lists for ieee 802.11 s-based smart grid ami networks[J]. Smart Grid, IEEE
Transactions on, 6(5): 2366-2374.

[8] Huang S C, Lu C N, Lo Y L. (2015): Evaluation of AMI and SCADA Data Synergy for Distribution Feeder Modeling[J]. Smart Grid, IEEE Transactions on, 6(4): 1639-1647.

[9] Bhatia R K, Bodade V. (2014): Defining the framework for wireless-AMI security in smart grid[C]//Green Computing Communication and Electrical Engineering (ICGCCEE), 2014 International Conference on. IEEE, 1-5.

[10] Park K Y, Yong S K, Kim J. (2012): Security enhanced IEEE $802.1 \mathrm{x}$ authentication method for WLAN mobile router[C]//Advanced Communication Technology (ICACT), 2012 14th International Conference on. IEEE, 549-553.

[11] Chi K H, Shih Y C, Liu H H, et al. (2011): Fast Handoff in Secure IEEE 802.11s Mesh Networks[J]. Vehicular Technology IEEE Transactions on, 60(1):219-232.

[12] Yuan G, Wen H, Zhang H F, et al. (2014): Efficient routing strategy on scale-free network based on local information[C]//IEEE International Conference on Software Engineering \& Service Science. IEEE, 11261129.

[13] Zhu K, Li W, Fu X.(2014): SMART: A social-and mobile-aware routing strategy for disruption-tolerant networks[J]. Vehicular Technology, IEEE Transactions on, 63(7): 3423-3434.

[14] Addis B, Capone A, Carello G, et al. (2014): Energy management through optimized routing and device powering for greener communication networks $[\mathrm{J}]$. IEEE/ACM Transactions on Networking (TONNE), 22(1): 313-325.

[15] Rifkin J. (2013): The third industrial revolution : how lateral power is transforming energy, the economy, and the world[M]. Palgrave Macmillan.

[16] Sun Q, Han R, Zhang H, et al. (2015): A multiagentbased consensus algorithm for distributed coordinated control of distributed generators in the energy internet[J]. Smart Grid, IEEE Transactions on, 6(6): 3006-3019.

[17] Zhou X, Wang F, Ma Y. (2015): An overview on energy internet[C]//Mechatronics and Automation (ICMA), 2015 IEEE International Conference on. IEEE, 126-131.

[18] Barabasi A L, Albert R. (1999): Emergence of Scaling in Random Networks[J]. Science, 286(5439):509-512.

[19] Ming T, Tao Z. (2011): Efficient routing strategies in scale-free networks with limited bandwidth.[J]. Physical Review E, 84(2):1855-1866.

[20] Jiang Z Y, Liang M G. (2013): Incremental routing strategy on scale-free networks[J]. Physica A: Statistical Mechanics and its Applications, 392(8): 1894-1901.

Printed by Pradip Kumar Chanda at The Indian Press Pvt. Ltd. 93A Lenin Sarani, Kolkata 700013 and published by him for Books \& Journals Pvt. Ltd. from 62 Lenin Sarani, Kolkata 700013 